Gastroenterologe 2017 · 12:38-48 DOI 10.1007/s11377-016-0127-z Online publiziert: 3. Januar 2017 (c) Der/die Autor(en) 2016. Dieser Artikel ist eine Open-Access-Publikation.

\section{Redaktion}

J. Hampe, Dresden

S. Schreiber, Kiel

CrossMark

F. Degenhardt · A. Franke

Institut für Klinische Molekularbiologie, Christian-Albrechts-Universität zu Kiel, Kiel, Deutschland

\title{
Genetik des Morbus Crohn und der Colitis ulcerosa
}

\section{Aktueller Stand 15 Jahre nach Entdeckung von NOD2}

\begin{abstract}
Die beiden häufigsten chronischentzündlichen Darmerkrankungen (CED) sind Morbus Crohn und Colitis ulcerosa. Die meisten CED-Patienten besitzen eine genetische Veranlagung für ihre Erkrankung und sind Träger von mehreren genetischen Risikovarianten. Was seit dem Jahr 2001, in dem das für Morbus Crohn bedeutsamste Krankheitsgen NOD2 entdeckt wurde, in der genetischen Forschung passiert ist und welche klinische Bedeutung den neuen Erkenntnissen beigemessen werden kann, ist im Folgenden zusammengefasst. Der Schwerpunkt liegt dabei vor allem auf der prägnanten Zusammenfassung großer multizentrischer Studien in europäischstämmigen Patienten mit entsprechender statistischer Signifikanz.
\end{abstract}

\section{Epidemiologie}

Über eine Million US-Amerikaner und 2,5 Millionen Europäer leiden unter CED, mit steigender Inzidenz auch außerhalb des europäischen Kontinents (z. B. Asien und dem mittleren Osten) sowie Südamerika. Damit wird die Erkrankung zunehmend zu einem globalen Problem und einer immer größeren Last für das Gesundheitssystem [18]. Trotz intensiver Forschung gilt CED noch immer als eine idiopathische Erkrankung, was bedeutet, dass man zwar viele Risikofaktoren kennt, aber die Pathologie noch nicht vollständig erklären kann. Die CED sind multifaktorielle polygene Erkrankungen und lassen sich entsprechend nicht auf eine einzelne Ursache zurückführen: Es handelt sich um eine Verbindung von genetischer Veranlagung, noch näher zu bestimmenden Risiko- und Umweltfaktoren (etwa Rauchen, Ernährungsgewohnheiten, Hygiene) und einer Störung der Barrierefunktion der Darmschleimhaut, sodass Darmbakterien sich auf der Darmwand ansiedeln und eine starke Immunreaktion auslösen können. Auch die Zusammensetzung des Darmmikrobioms (die Gesamtheit aller Bakterien, Viren, Pilze, Protozoen und Archaeen im Darm) spielt eine wichtige Bedeutung bei der Krankheitsentstehung. Ein krankheitsauslösendes Pathogen konnte bis heute nicht eindeutig identifiziert werden, besonders das Mycobacterium avium subsp. paratuberculosis (MAP) wurde in der Vergangenheit (zu Unrecht) als Krankheitsauslöser verdächtigt [9].

Psychische Ursachen werden derzeit als Krankheitsauslöser ausgeschlossen, anerkannt ist aber, dass sich psychische Faktoren auf den Verlauf der Erkrankung auswirken können. Immer mehr aktuelle Studien zeigen auch eine Verbindung zwischen psychischen Faktoren und der Zusammensetzung des Darmmikrobioms („gut-brain axis").

\section{》) Epidemiologische Studien implizieren die Existenz geneti- scher Suszeptibilitätsfaktoren}

Epidemiologische Studien haben eine familiäre Häufung von CED-Patienten gezeigt: $2-14 \%$ der Patienten mit Morbus Crohn haben mindestens einen
Verwandten, der ebenfalls an Morbus Crohn erkrankt ist [13]. Sie implizieren damit die Existenz genetischer Suszeptibilitätsfaktoren. Eine Metaanalyse von 6 Zwillingsstudien lieferte im Jahr 2011 Konkordanzraten für Morbus Crohn von $30,3 \%$ für eineiige (d.h. nahezu $100 \%$ genetisch identisch) und 3,6\% für zweieiige Zwillinge [10]. Zwar überschätzen Zwillingsstudien gegebenenfalls die Vererbbarkeit (Heritabilität) - eine ganz aktuelle Studie zeigt, dass „familiäre Umweltfaktoren" dringend berücksichtig werden müssen [24] - jedoch fehlt vielen Zwillingsstudien auch ein mehrjähriges Follow-up, d.h. man unterschätzt die Zahl der neuerkrankten Zwillingspartner aufgrund der Momentaufnahme der einmal erhobenen Daten. Zusammenfassend lässt sich aber mit großer Sicherheit sagen, dass die CEDPathogenese eine signifikante genetische Komponente hat, jedoch CED (abgesehen von den seltenen frühkindlichen Formen) keine Erbkrankheiten im klassischen Sinne sind.

\section{Entdeckung von NOD2}

Im Jahr 1996 lieferte eine Kopplungsanalyse den ersten Hinweis auf ein CEDKrankheitsgen auf Chromosom 16 [15]. Im Jahr 2001 wurden dann 3 Risikovarianten für Morbus Crohn in NOD2 aufChromosom 16 identifiziert (R702W, G908R und L1007 fs; [16]). Das OddsRatio für die Varianten wurde mit 2-4 für heterozygote Träger und 20-40 für homozygote Träger beschrieben. Mindestens eine dieser Varianten liegt bei 


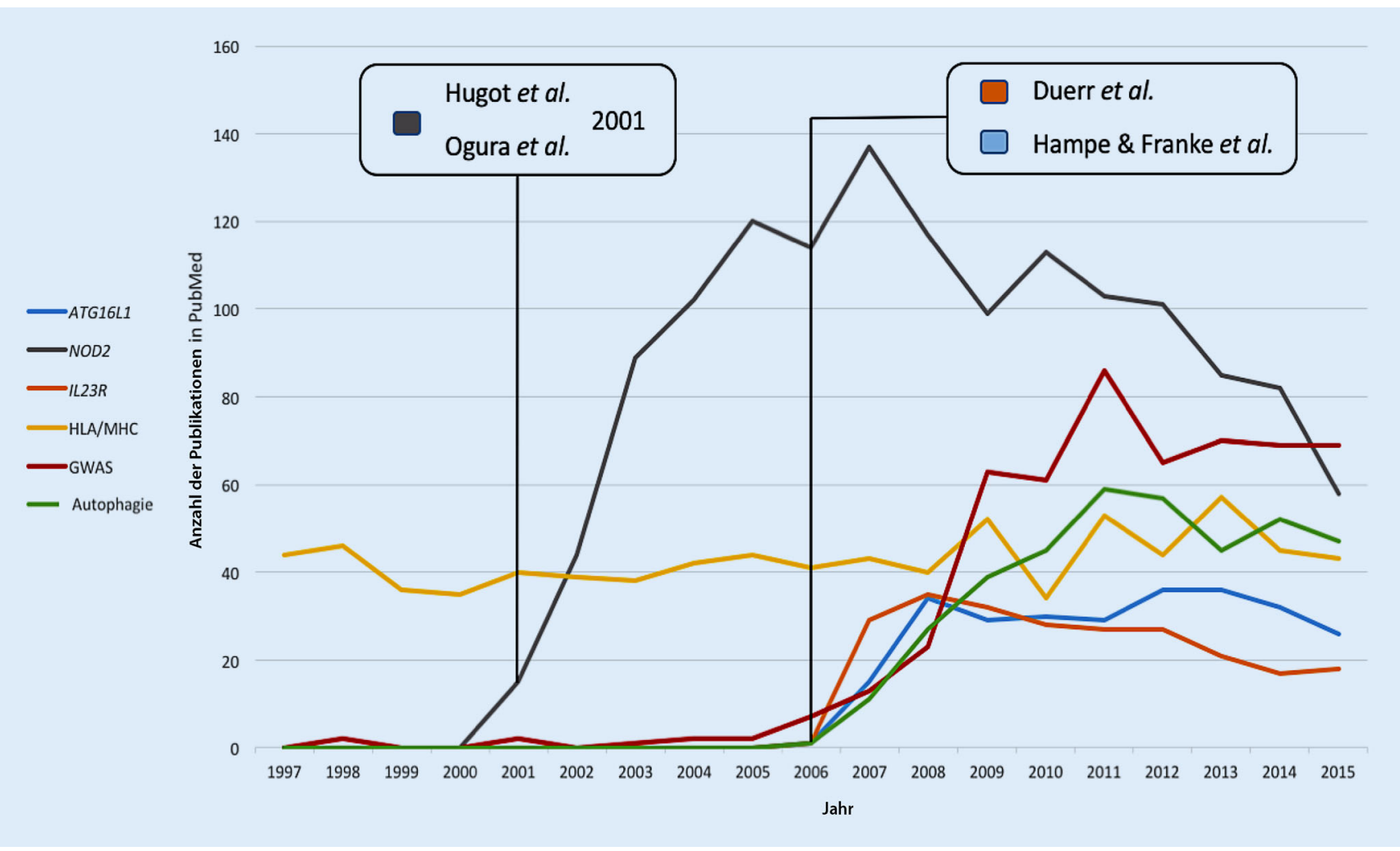

Abb. 1 ॥ Genidentifikationen führen zu zahlreichen weiterführenden Forschungsarbeiten zum entsprechenden Kandidatengen, dessen Protein und Stoffwechselweg. Die Y-Achse zeigt die Anzahl der in PubMed gelisteten Artikel für chronischentzündliche Darmerkrankungen (CED; oder Morbus Crohn oder Colitis ulcerosa) und die entsprechenden bekannten Kandidatengene. Vor der Identifikation von ATG16L1 als Risikogen für Morbus Crohn wurde z. B. der Stoffwechselweg Autophagie im Kontext der CED noch nicht untersucht, danach ist ein nahezu exponentieller Anstieg zu erkennen (grüne Linie). GWAS genomweite Assoziationsstudie

30-40 \% der Patienten mit Morbus Crohn vor, verglichen mit 6-7\% bei gesunden Europäern [22]. In den Jahren 1996-2004 wurden insgesamt 11 Kopplungsanalysen für Morbus Crohn publiziert (Review in [22], Metaanalyse: [31]). Zusammenfassend waren die Ergebnisse der Kopplungsanalysen eher enttäuschend, nur wenige robuste Signale wurden neben NOD2 identifiziert.

\section{》) NOD2 ist das am besten validierte Krankheitsgen für Morbus Crohn}

Schon im Jahr 1996 zeigten Risch et al. [27], dass 18.000 betroffene Geschwisterpaare in einer Kopplungsanalyse benötigt würden, um Odds-Ratios größer als 1,5 für Varianten mit einer Allelfrequenz von 50 \% zu identifizieren. Es gilt: Je höher die Allelfrequenz und je größer das OddsRatio, d. h. die Effektstärke, desto größer ist die statistische Power, eine Risikovariante zu identifizieren. Nach wie vor ist NOD2 das am besten untersuchte und am besten validierte Krankheitsgen für Morbus Crohn: Für die Ätiologie der Colitis ulcerosa sind NOD2-Risikovarianten sehr wahrscheinlich nicht relevant.

\section{Genomweite Assoziationsstudien}

Umfangreiche "genetische Landkarten“, die über eine Million „single nucleotide polymorphisms“ (Einzelnukleotidpolymorphismen, SNPs) im menschlichen Genom katalogisierten, und die Etablierung der Chiptechnologie in der Biomedizin machten schließlich genomweite Assoziationsstudien (GWAS) mit kommerziellen SNP-Arrays und über 500.000 zu testenden SNPs pro Probe möglich. Typischerweise werden dabei in einem GWAS-Ansatz DNA-Proben von mindestens 2000 nicht verwandten Patienten und 3000 Kontrollpersonen untersuch. Einige der wichtigsten Studien und Befunde sind in $\bullet$ Tab. 1 zusammengefasst. Die nunmehr 10 Jahre andauernde sogenannte „GWAS-Ära“ lässt sich als äußerst erfolgreiches Kapitel der CED-Genetikforschung zusammenfassen. War bis zum Jahr 2001 noch kein Krankheitsgen für die CED bekannt, wurden mittlerweile dank GWAS über 200 Krankheitsloci beschrieben. Die Beschreibung der assoziierten Stoffwechselwege hat das Krankheitsverständnis der CED enorm verbessert. Abb. 1 zeigt exemplarisch, dass die Identifikation eines einzelnen Krankheitsgens - und im konkreten Fall eines neuen Stoffwechselwegs - eine regelrechte „Forschungslawine“ lostreten kann.

Gezielte Studien, z. B. zur Autophagie im Mausmodell, wurden erst nach Identifikation des Kandidatengens ATG16L1 möglich und lieferten zusätzliche mechanistische Hinweise zur Relevanz dieses Stoffwechselwegs. 
Gastroenterologe 2017 · 12:38-48 DOI 10.1007/s11377-016-0127-z

(c) Der/die Autor(en) 2016. Dieser Artikel ist eine Open-Access-Publikation.

\section{F. Degenhardt · A. Franke}

\section{Genetik des Morbus Crohn und der Colitis ulcerosa. Aktueller Stand 15 Jahre nach Entdeckung von NOD2}

\section{Zusammenfassung}

Modernste Technologien der genetischen Forschung bieten der Medizin einen ganz neuen Zugangsweg zur Entdeckung von genetischen Krankheitsursachen. Innerhalb der letzten 15 Jahre wurden die genetischen Untersuchungstechniken in einem solchen Umfang weiterentwickelt, dass heute auch die Untersuchung "komplexer" Erkrankungen und die Entschlüsselung kompletter Patientengenome innerhalb kürzester Zeit möglich sind.

Für die chronisch-entzündlichen Darmerkrankungen (CED) Morbus Crohn und Colitis ulcerosa sind mittlerweile über 200 assoziierte Genloci bekannt. Die Mehrheit der identifizierten Loci überschneidet sich nicht nur zwischen Morbus Crohn und Colitis ulcerosa, was deren klinische Ähnlichkeit widerspiegelt, sondern auch mit anderen chronisch-entzündlichen Erkrankungen, vor allem mit Psoriasis, Morbus Bechterew und primären Immundefizienzen. Die beiden wichtigsten und am besten validierten CEDKrankheitsloci sind nach wie vor NOD2 (für Morbus Crohn) und die HLA-Region (für Colitis ulcerosa). Genetische Analysen in anderen Ethnizitäten (z. B. Asiaten) zeigen nahezu die gleichen assoziierten Risikoloci wie in europäischstämmigen Patienten. Interessanterweise ist NOD2 hier eine Ausnahme.

Die jüngsten großen genetischen Studien bestätigen die Assoziation von Risikovarianten in NOD2 mit Dünndarmbefall bei Morbus Crohn und suggerieren die Existenz von eher drei als zwei Subformen der CED: 1) Colitis ulcerosa, 2) Morbus Crohn mit Dickdarmbefall, 3) Morbus Crohn mit Dünndarmbefall. Durch hochmoderne Sequenzieranalysen konnten in den letzten Jahren für frühkindliche Formen der CED einzelne Mutationen vor allem in bekannten Immundefizienzgenen (neben den bekannten Mutationen in IL1ORA/B), identifiziert und die Erkrankungen damit aufgeklärt werden (monogener Defekt).

Schlüsselwörter

Morbus Crohn - Colitis Ulcerosa - Chronischentzündliche Darmerkrankungen · Genomweite Assoziationsstudie · Genetische Risikofaktoren · Humanes Leukozytenantigen . Genetische Pleiotropie

\section{Genetics of Crohn's disease and ulcerative colitis. Current status 15 years after discovery of NOD2}

\section{Abstract}

Modern technologies in genetic research hold the promise of identifying yet unknown causes of diseases, revealing the relevant pathways, and prioritizing therapeutic targets. Technological developments of the last 15 years tremendously contributed to novel genetic findings in complex disease research. Entire patient genomes can now be deciphered within a few days at reasonable and continuously decreasing costs. More than 200 genetic susceptibility loci have been identified for the inflammatory bowel (IBD) diseases, Crohn's disease (CD), and ulcerative colitis (UC). Reflecting their clinical similarity, most of the risk loci are shared between $C D$ and UC outside the major histocompatibility complex (MHC). The genetic risk map of IBD is also highly similar to other chronic immune-mediated diseases, especially psoriasis, ankylosing spondylitis, and primary immunedeficiencies. The two best validated IBD disease loci are still NOD2 for CD and the HLA/MHC region on chromosome 6p21 for UC. Genetic investigations in non-European-ancestry cohorts (e. g., from Eastern Asia) also suggest that risk loci are shared across different ancestries. Interestingly, this is not true for NOD2.

A recent large-scale and multicenter study validated the association of susceptibility variants within the NOD2 gene and ileal CD. These genetic analyses also support the theory that rather three than two subtypes of IBD exist: (1) UC, (2) colonic CD, and (3) ileal CD.
Modern high-throughput sequencing studies revealed several monogenic forms of early onset and very early onset IBD, implicating often known immunodeficiency disease loci, including the previously implicated interleukin-10 receptor (IL1ORA/B) gene. Thus, identifying a single causative genetic variant in these young patients proves their exact disease cause.

\section{Keywords}

Morbus Crohn · Colitis Ulcerosa · Inflammatory bowel diseases . Genome-wide association study - Genetic susceptibility . Major Histocompatibilty Complex · Genetic pleiotropy
Die durchschnittliche durch GWAS identifizierte CED-Risikovariante ist in der Normalbevölkerung häufig (mediane Allelfrequenz etwa $30 \%$ ) und besitzt ein niedriges Odds-Ratio (mediane Effektstärke von 1,1). Trotz der großen statistischen Signifikanz der Varianten bedeuten die hohen Allelfrequenzen und niedrigen Effektstärken einen geringen prädiktiven Wert (,area under receiver operator curve“, AUC, von 0,60 bei Un- terscheidung Morbus Crohn vs. Colitis ulcersosa; [5]). Und die Erfahrung belegt, was die Statistiker prognostiziert haben: Je mehr Fälle und Kontrollen (idealerweise zwei bis dreimal soviele Kontrollen wie Patienten) in eine GWAS eingeschlossen werden, desto größer ist die statistische Power und desto mehr signifikante Loci können genomweit identifiziert werden [32]. Die genomweite Signifikanz, ein $p$-Wert der kleiner als $5 \times 10^{-8}$ ist, gilt in der Genetik heutzutage als etabliertes Signifikanzniveau.

Im Jahr 2016 wurde die größte internationale genetische Assoziationsstudie für CED-Subphänotypen veröffentlicht [5]. Genetische Daten und Patientendaten aus 49Zentren und 16Ländern (Europa, Nordamerika und Australasien) wurden bezüglich Genotyp-Phänotyp-Assoziationen untersucht. Insgesamt wurden 16.902 Patienten mit Morbus Crohn und 


\begin{tabular}{|c|c|c|}
\hline Jahr & Studie & Ergebnisse (Beispiele) \\
\hline 2005 & Yamazaki et al. [34] & $\begin{array}{l}\text { Erstidentifikation von TNFSF15, spezifisches Krankheitsgen } \\
\text { für Asiaten }\end{array}$ \\
\hline 2006 & Duerr et al. [6] & Erstidentifikation von IL23R \\
\hline 2007 & $\begin{array}{l}\text { Hampe \& Franke et al. } \\
\text { [14] }\end{array}$ & $\begin{array}{l}\text { Erstidentifikation von ATG16L1 und Implikation des Autopha- } \\
\text { giestoffwechselwegs bei CED }\end{array}$ \\
\hline 2007 & WTCCC [33] & $\begin{array}{l}11 \text { Loci für Morbus Crohn, davon } 4 \text { neu (z. B. Autophagiegen } \\
\text { IRGM) }\end{array}$ \\
\hline 2008 & Barrett et al. [2] & $\begin{array}{l}\text { Metaanalyse für Morbus Crohn, Identifikation von } 32 \text { Loci } \\
\text { (davon } 21 \text { neu) }\end{array}$ \\
\hline 2008 & Franke et al. [10] & $\begin{array}{l}\text { Erste GWAS für Colitis ulcerosa, Identifikation von } 4 \text { neuen } \\
\text { Loci inklusive IL } 10\end{array}$ \\
\hline 2010 & McGovern et al. [23] & Metaanalyse für Morbus Crohn, Identifikation von FUT2 \\
\hline 2010 & Franke et al. [11] & $\begin{array}{l}\text { Metaanalyse für Morbus Crohn, Identifikation von } 71 \text { Loci } \\
\text { (davon } 30 \text { neu) }\end{array}$ \\
\hline 2011 & Anderson et al. [1] & $\begin{array}{l}\text { Metaanalyse für Colitis ulcerosa, Identifikation von } 47 \text { Loci } \\
\text { (davon } 29 \text { neu) }\end{array}$ \\
\hline 2012 & Jostins et al. [17] & $\begin{array}{l}\text { Genomweite Kandidatengenanalyse (Immunochip), Identifi- } \\
\text { kation von } 163 \text { Loci für CED (davon } 71 \text { neu) }\end{array}$ \\
\hline 2015 & Liu et al. [21] & $\begin{array}{l}\text { Metaanalyse (Immunochip) zusammen mit knapp } 10.000 \\
\text { Patienten aus Ostasien, Indien und dem Iran; Identifikation } \\
\text { von } 38 \text { neuen Loci }\end{array}$ \\
\hline 2016 & Ellinghaus et al. [7] & $\begin{array}{l}\text { Metaanalyse/phänotypübergreifende Analyse (Immunochip) } \\
\text { von } 5 \text { verschiedenen Erkrankungen: Morbus Crohn, Colitis } \\
\text { ulcerosa, Morbus Bechterew, Psoriasis und primär sklerosie- } \\
\text { rende Cholangitis (PSC); } 244 \text { Loci identifiziert, davon } 27 \text { neu } \\
\text { (und davon wiederum } 6 \text { neu für Morbus Crohn) }\end{array}$ \\
\hline \multicolumn{3}{|c|}{$\begin{array}{l}\text { `Eine detailliertere und vollständigere Aufstellung ist der Tab. } 1 \text { in Ellinghaus et al. [7] zu entnehmen. } \\
\text { CED chronisch-entzündliche Darmerkrankungen }\end{array}$} \\
\hline
\end{tabular}

12.597 Patienten mit Colitis ulcerosa in die Analysen eingeschlossen. Es konnten keine signifikanten genetischen Assoziationen mit dem Krankheitsverlauf oder Komplikationen gefunden werden. Eine starke Assoziation mit einem frühen Ersterkrankungsalter zeigten 3 Loci: 3p21 (MST1), NOD2 und HLA. NOD2 zeigte außerdem eine starke Assoziation mit ,ilealem Befall“ (aber nicht mit Befall des Dickdarms und höherem Schweregrad/Komplikationen wie vorher angenommen). Die wichtigste klinische Erkenntnis aus den genetischen Analysen der Studie war, dass die CED nicht mehr binär in Morbus Crohn und Colitis ulcerosa unterteilt werden sollten, sondern ein kontinuierliches Spektrum von Erkrankungen mit Colitis ulcerosa und ilealem Morbus Crohn an den entgegengesetzten Polen darstellen.

Morbus Crohn mit Dickdarmbefall ist dabei der Colitis ulcerosa auf molekularer Ebene ähnlicher, wie ileokolischer Morbus Crohn dem ilealen Morbus Crohn nä- humanes Leukozytenantigen). Die HLARegion spielt bei fast allen chronisch-entzündlichen Erkrankungen die wichtigste Rolle, hier liegen meist die signifikantesten Risikovarianten mit den größten Effektstärken. Bei den CED sind vor allem die klassischen HLA-Gene am stärksten assoziiert, wobei die Assoziation für die Colitis ulcerosa deutlich stärker ist als für den Morbus Crohn (hier Klasse I stärker assoziiert als bei Colitis ulcerosa). Für beide Erkrankungen ist das Klasse-II-Gen DRB1 der am stärksten assoziierte Genort [12]. Allerdings ist die Assoziation am HLA-Lokus nach wie vor äußerst komplex und lässt sich aufgrund der großen Dichte an genetischen Varianten/Kandidatengenen und der starken Kopplung (große Chromosomenabschnitte werden während der Meiose zwischen väterlichen und mütterlichen Chromosomen nicht „gemischt") genetisch nur schwer auflösen.

\section{》) HLA-DRB ${ }^{*} 01: 03$ ist das am stärksten assoziierte HLA-Allel für Colitis ulcerosa}

Das am stärksten assoziierte HLA-Allel für Colitis ulcerosa ist $H L A-D R B 1^{\star} 01: 03$ (Odds-Ratio $=3,59 ; p=3 \times 10^{-19}$ ), das Klasse-II-Antigen-präsentierende Zellen (dendritische Zellen, Makrophagen, B-Zellen) und damit CD4-positive T-Zellen pathophysiologisch impliziert. Große funktionelle genomische Studien, die Genexpressions- und Epigenetikdaten von verschiedenen Zelltypen mit genetischen Informationen integriert analysierten, haben parallel CD4-positive Zellen (v.a. $\mathrm{T}_{\mathrm{H}} 0$ ) als wichtigsten Zelltyp bei der Colitis ulcerosa identifiziert [8]. Eine offene Frage ist, ob ein exogenes Antigen bei der Colitis ulcerosa eine zentrale Rolle spielt oder welches das genaue körpereigene Antigen ist.

\section{Krankheitsübergreifende Studien}

Wie im Ausblick nachfolgendgeschildert gibt es eine signifikante genetische Schnittmenge zwischen den CED und der klassischen Infektionserkrankung Lepra. Über die Hälfte der bekann- 


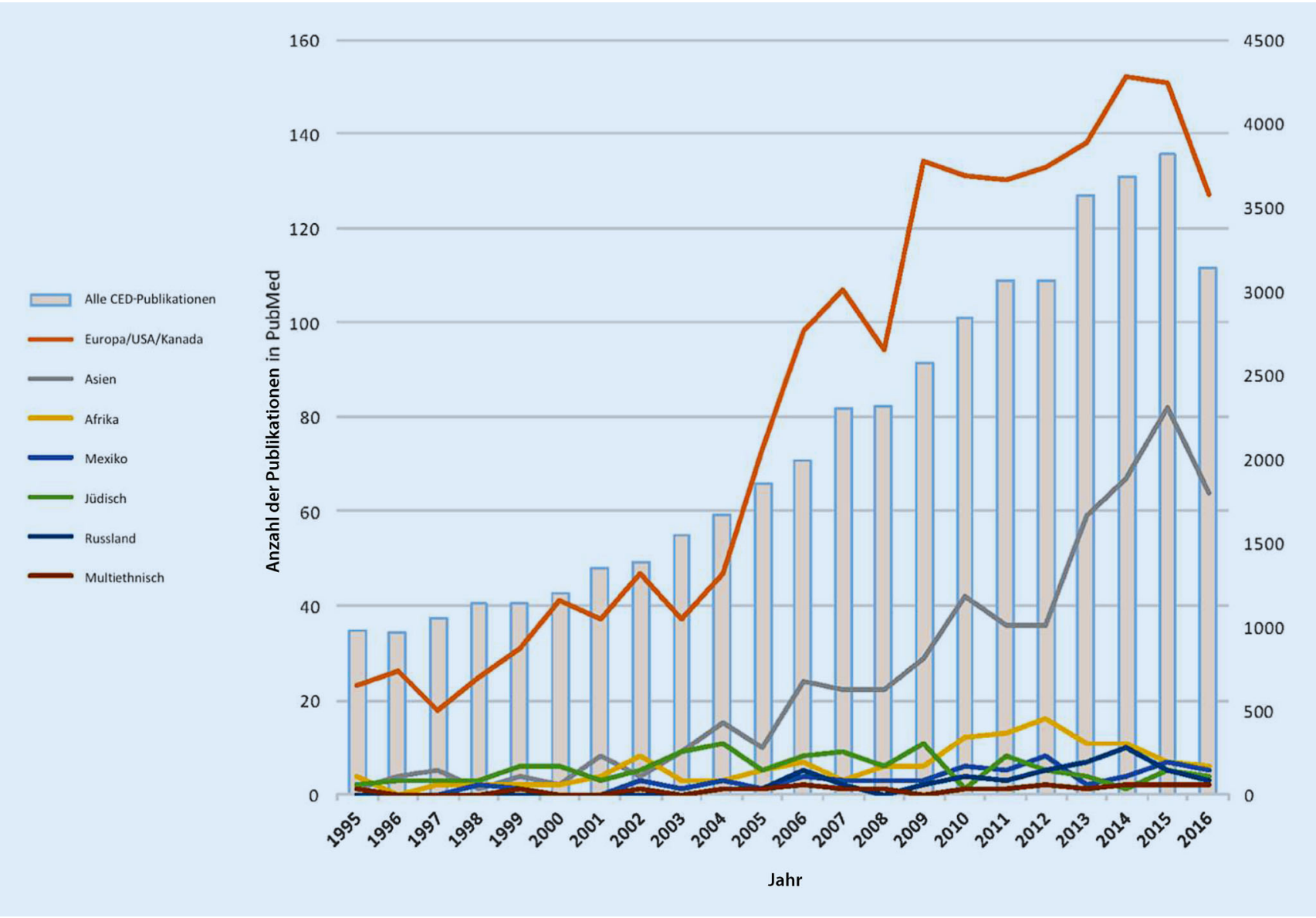

Abb. 2 \ Studien über chronisch-entzündliche Darmerkrankungen (CED) in nichteuropäischstämmigen Ethnizitäten sind nach wie vor unterrepräsentiert. Klar zu erkennen ist ein steiler Anstieg der CED-Veröffentlichungen seit dem Jahr 2003, vor allem für europäischstämmige Patienten (orange Linie). Seit etwa 2005 ist ein steiler Anstieg für Untersuchungen in asiatischen Kohorten (graue Linie) zu verzeichnen. Die linke Y-Achse zeigt die Anzahl der CED-Publikationen pro Jahr für die genannte Ethnie, die rechte Y-Achse zeigt die Anzahl der gesamten CED-Publikationen in PubMed pro Jahr (Balkendiagramm im Hintergrund)

ten CED-Risikoloci überlappen mit bekannten Risikoloci für andere chronisch-entzündliche Erkrankungen [17]. $\mathrm{Ob}$ es letztlich auch die gleichen Gene oder vielleicht sogar die gleichen Varianten mit gleichen Effektrichtungen sind, lässt sich nur durch aufwändige Feinkartierungsstudien und systematische krankheitsübergreifende Analysen herausfinden. Ellinghaus et al. (siehe - Tab. 1) haben solch eine systematische Analyse im Jahr 2016 veröffentlicht. Durch die kombinierte Analyse von 5 verschiedenen Erkrankungen, inklusive Morbus Crohn und Colitis ulcerosa, konnten Ellinghaus et al. zeigen, dass in der Tat eine große genetische Ähnlichkeit zwischen den genannten 5 Erkrankungen besteht. Allerdings sind die Beziehungen komplexer als zuvor angenommen und teilweise unterscheiden sich die
Effektrichtungen und -stärken der einzelnen Varianten stark. Oft sind es auch verschiedene Varianten am gleichen Locus, die bei der anderen Erkrankung assoziiert sind.

Die Studie kommt zu dem Schluss, dass genetische Pleiotropie - und nicht die klinische Heterogenität der Erkrankungen (z. B. exzessive Komorbidität) die primäre Erklärung für die molekulare Ähnlichkeit verschiedener Erkrankungen ist. Genetische Pleiotropie bedeutet, dass ein einzelnes Gen (oder eine einzelne Variante) mehrere phänotypische Merkmale ausprägen kann. Eine Analyse sämtlicher elektronischer Patientenakten in Dänemark aus den letzten Jahren bestätigte, dass die 5 untersuchten Erkrankungen hochsignifikant miteinander assoziiert sind und häufig im gleichen $\mathrm{Pa}$ tienten auftreten $\left(p<1,21 \times 10^{-9}\right.$ für 10 der 12 möglichen Krankheitskombinationen). Für weitere Informationen zum Thema werden die Übersichtsartikel von Lees et al. [20] beziehungsweise Parkes et al. [26] empfohlen.

\section{Genetische Studien in anderen Ethnien}

- Abb. 2 veranschaulicht, dass der überwiegende Teil an CED-Studien aus Europa oder Nordamerika kommt. Jährlich werden nur wenige CED-Studien $\mathrm{zu}$ ethnischen Minderheiten in Nordamerika (Asiaten, Mexikaner, Afroamerikaner) oder aus den entsprechenden Heimatländern publiziert. Erfreulich ist generell insgesamt der große Anstieg an CED-Fachartikeln pro Jahr seit 2003 und die immer stärker werdende CED-Forschung in Asien (linearer 


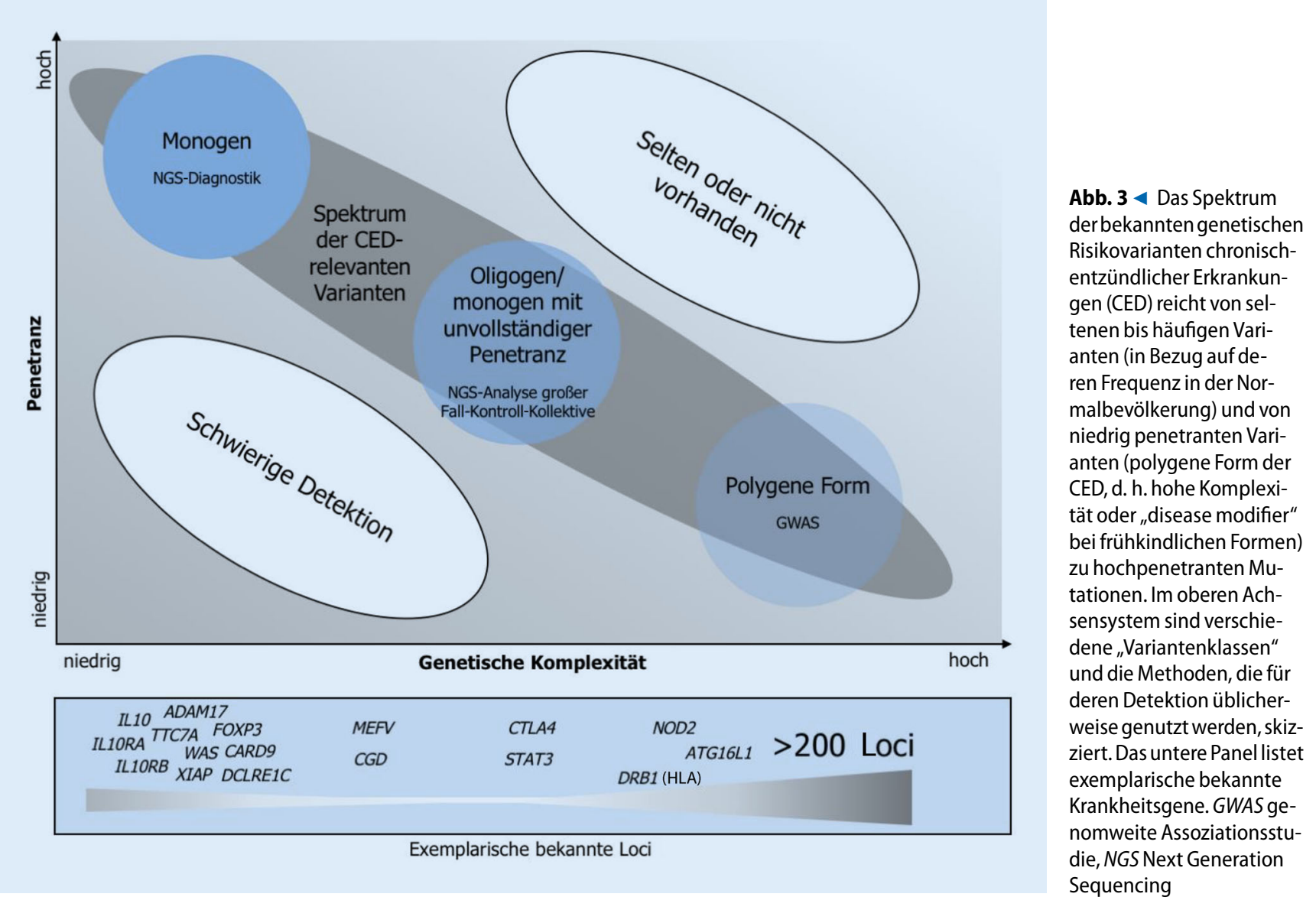

Anstieg seit 2005). Dieser Anstieg lässt sich wahrscheinlich auch durch die steigende CED-Inzidenz und -Prävalenz in Asien und die damit verbundene Bedeutung für das Gesundheitssystem und das öffentliche Leben erklären. Eine der größten genetischen Studien zu CED für Ostasien (Japan, Korea, Hong-Kong-Chinesen), Indien und den Iran (siehe - Tab. 1, [21]) zeigte, dass die Effektstärken und -richtungen für die bekannten CED-Risikoloci nahezu identisch mit den Daten von europäischstämmigen Patienten sind. Nur wenige Loci zeigten signifikante Unterschiede in den Ostasiaten (NOD2 $\rightarrow$ in Ostasien nicht relevant, HLA $\rightarrow$ signifikante Unterschiede bei den assoziierten Allelen/Genen, TNFSF15 $\rightarrow$ in Ostasiaten deutlich relevanter). Unterschiede in Umwelteinflüssen, Ernährungsverhalten, genetischer Abstammung (und in entsprechend anderer evolutionärer Historie) und menschlicher Physiologie erklären wahrscheinlich die wenigen, aber signifikanten Unterschiede zwischen
Ostasiaten und europäischstämmigen Patienten.

\section{Frühkindliche Formen chronisch-entzündlicher Darmerkrankungen}

Die meisten CED-Patienten haben eine komplexe polygene Form der Erkrankung, d.h. mehrere genetische Risikovarianten im Zusammenspiel mit pathophysiologisch deutlich relevanteren (unbekannten) Umweltfaktoren führen zur Erkrankung (siehe - Abb. 3). In den letzten Jahren - und seit dem ersten Artikel von Glocker et al. zum Thema im The New England Journal of Medicine im Jahr 2009 - sind zunehmend Berichte über Patienten mit monogenen beziehungsweise oligogenen Erkrankungsformen der CED erschienen. Hierbei handelt es sich überwiegend um frühkindliche äußerst schwere Formen der Erkrankung mit Erstmanifestation in den ersten 8 Jahren, jedoch auch in einzelne Fälle bis zum 16. Lebensjahre. Bahnbre- chende Entwicklungen in der Sequenziertechnologie - ein menschliches Genom kann heute innerhalb kürzester Zeit in einem Labor vollständig entschlüsselt werden - ebneten den Weg für die einfachere Aufklärung von monogenen Mendel-Erkrankungen. Die genetischen Defekte dieser CED-ähnlichen monogenen Erkrankungen beeinträchtigen meist die intestinale Barrierefunktion oder sind ursächlich für einen Immundefekt (d. h. gestörte Granulozyten- und Phagozytenaktivität, hyper- und autoinflammatorische Störungen oder gestörte B- sowie T-Lymphozyten-Funktionen). Einen detaillierten und aktuellen Überblick liefert der Übersichtsartikel von Uhlig et al. [30]. Die Identifikation einzelner Gene und Mutationen hat das Verständnis der Pathogenese von CED enorm verbessert und erlaubt in einigen Fällen auch die exakte Klärung der Krankheitsursachen. Seit dem 1.7.2016 ist eine Genpaneldiagnostik mit Next Generation Sequencing (NGS) auch gemäß einheitlichem Bewertungsmaßstab (EBM) abrechenbar 


\section{Infobox 1 Weiterführende internetbasierte Informationen}

- International Inflammatory Bowel Disease Genetics Consortium (IIBDGC). https:// www.ibdgenetics.org. Zugegriffen: 6 . Dezember 2016

- Kassenärztliche Bundesvereinigung. Praxisnachrichten. Weiterentwicklung der Gebührenordnungspositionen zum 1. Juli. http://www.kbv.de/html/1150_21752. php. Zugegriffen: 6. Dezember 2016

(- Infobox 1) und sollte bei Verdacht auf eine frühkindliche schwere CED-ähnliche Erkrankung entsprechend (über ein Institut für Humangenetik und Speziallabore) beauftragt werden. Die Identifikation eines entsprechenden Immundefekts kann neue therapeutische Optionen in therapierefraktären Patienten eröffnen. Entscheidend ist das Ersterkrankungsalter; ältere Patienten mit frühem Ersterkrankungsalter können natürlich bei entsprechendem Verdacht auch einem diagnostischen NGS-Test unterzogen werden.

\section{Ausblick}

Moderne genetische Analysen sind äußerst exakt, quantitativ (Allelzahlen können sehr präzise gemessen werden) und liefern extrem hochaufgelöste Daten des menschlichen Genoms. Die Identifikation von über 200 Krankheitsvarianten für die CED und die Aufklärung diverser frühkindlicher Formen der CED haben das Verständnis dieser Erkrankung enorm verbessert. Über 1444 Kandidatengene liegen in 163 der 200 CEDassoziierten genomischen Regionen. Von diesen konnten Jostins et al. [17] 300 Gene als wahrscheinlich krankheitsrelevant mithilfe bioinformatischer Verfahren priorisieren. Abgesehen von NOD2, ATG16L1, IL23R, IL10 und anderen wenigen Ausnahmen ist bis jetzt also unklar, welches die eigentlichen Risikogene in den assoziierten genomischen Regionen und letztlich welche genomischen Varianten relevant sind: Sind es häufige oder seltene kausative Varianten? Welche Art von Varianten, d. h. SNPs, „singlenucleotidevariants“
(SNVs), Insertionen/Deletionen oder Kopienzahlvariationen, liegen vor?

Weiterführende Untersuchungen bis hin zu Studien im Tiermodell sind nötig, um die genaue Rolle der Kandidatengene zu untersuchen. Systematische Sequenzierexperimente in mehreren $10.000 \mathrm{~Pa}$ tienten sind außerdem nötwendig, um auch die selteneren genetischen Varianten (Allelfrequenz $<5 \%$ und v. a. $<1 \%$ in der Normalbevölkerung) auf Assoziation mit CED zu testen. Alle genetischen Befunde für die polygene Form der CED erklären bisher nur etwa 13,6 \% der phänotypischen Varianz für Morbus Crohn und 7,5 \% für Colitis ulcerosa [17]. Einen großen Anteil hat dabei die MHC-Region. Erklärt für Morbus Crohn der MHCIndex-SNP (dies ist der am stärksten assoziierte SNP in der Region) rs9264942 noch $0,3 \%$ der Varianz, sind es für alle HLA-Allele zusammen 3,1\%. Für Colitis ulcerosa ergeben sich 2,3\% der Varianz durch den Index-SNP rs6927022 und $6,2 \%$ durch alle HLA-Allele [12]. Diese Zahlen zeigen, dass abgesehen vom Rauchen, das ein etablierter Risikofaktor für Morbus Crohn ist, vor allem bislang noch nicht identifizierte Umweltfaktoren einen großen Anteil an der CED-Ätiologie haben müssen.

\section{》) Bislang nicht identifizierte Umweltfaktoren müssen einen großen Anteil an der CED- Ätiologie haben}

Die bisher identifizierten genetischen Risikofaktoren können natürlich Hinweise auf entsprechende Umweltfaktoren liefern, wie z. B. NOD2 das angeborene Immunsystem im Darmepithel (und die Rolle der Bakterien im Darm) auf das Tableau gebracht hat.

Noch größere Anstrengungen werden nötig sein, um nichtgenetische vererbbare molekulare Faktoren zu untersuchen. Jüngste Analysen konnten z. B. zeigen, dass eine chronische Darmentzündung im Mausmodell [29] auch Auswirkungen auf die nachfolgenden Generationen hat $\left(F_{1}-\right.$ und $F_{2}$-Generation) und Störungen der Genexpression im Darmepithel hervorruft. Epigenetische Verände- rungen der Keimbahn-DNA, z. B. DNAMethylierung, sind demnach als nichtgenetische Erbfaktoren zu berücksichtigen.

Nach Einschätzung der Autoren liegt das größte Potenzial, zumindest für die Colitis ulcerosa, in der Erforschung der HLA-Region auf Chromosom 6p21 und in gezielten immunogenetischen Analysen. Die HLA-Assoziation der Colitis ulcerosa kann mit der HLA-Assoziation bei Zöliakie verglichen werden (MHC-Klasse-II-Signal, hochsignifikante Assoziation; [19]). Bei der Zöliakie kennt man das Antigen "Gluten“ als wesentlichen krankeitsverursachenden Umweltfaktor. Es könnte sich also lohnen, ein entsprechendes Antigen bei der Colitis ulcerosa zu suchen.

Proteine in der „modernen“ Ernährung sowie des Darmmikrobioms erscheinen hier als plausible Testkandidaten. Das Zusammenspiel zwischen Ernährungsfaktoren, Darmmikrobiom und Wirt (hier: Gesamtheit der genetischen Varianten eines Menschen) ist Gegenstand zahlreicher laufender Untersuchungen und wird in den nächsten Jahren mit großer Wahrscheinlichkeit viele neue Erkenntnisse zur CED-Ätiologie liefern. In diesem Zusammenhang ist auch die Tatsache interessant, dass Varianten am NOD2-Genort, allerdings andere als die CED-Risikovarianten, die Hauptrisikovarianten für Lepra, eine klassische Infektionserkrankung ( $\mathrm{Myco}$ bacterium leprae), sind. Dieses konnte im Jahr 2010 in einer chinesischen GWAS für Lepra gezeigt werden [35]. Die Liste der Risikoloci für Lepra und CED überschneidet sich nahezu perfekt. Neben NOD2 wurden die bekannten CEDLoci C13orf31, RIPK2, TNFSF15 und die HLA-Region als Risikofaktoren für Lepra identifiziert. Damit ist die Mykobakterieninfektionshypothese für die CED wieder aktueller denn je. Wahrscheinlich ist jedoch, dass nicht nur Mykobakterien, sondern auch andere Bakterien des Mikrobioms eine entscheidende Rolle bei der Krankheitsentstehung spielen. Zusammenfassend könnte die Pathogenese der CED wie folgt verlaufen: Eine beeinträchtigte Darmbarriere (gestörtes Mikrobiom mit geringerer Artenvielfalt und mehr "schlechten“ als "guten“ Bakterien, Stress, Antibiotika, für die 
Hier steht eine Anzeige.

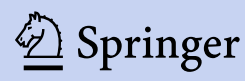


Mukosa toxische Ernährungsbestandteile) erleichtert Bakterien eine Ansiedelung auf dem Darmepithel. Die Barriere ist aufgrund genetischer Suszeptibilität durchlässiger (z. B. Störung der „tight junctions") und das angeborene Immunsystem geschwächt (z. B. NOD2-Defekt, Autophagiedefekt). Somit kann der Darminhalt leichter mit Zellen des adaptiven Immunsystems (z. B. mit dendritischen Zellen) in Berührung kommen. Genetische Suszeptibilität (die meisten der CED-relevanten Risikogene sind in Immunzellen exprimiert), mangelndes „immunologisches Training“ im frühen Kindesalter (Hygienehypothese) und die große „Dosis" an Bakterien und sonstigem Darminhalt hinter der Barriere führen zu einer übersteigerten Immunantwort. Diese Immunreaktion kann in genetisch suszeptiblen Personen auch nur langsam wieder herunterreguliert werden (z. B. Defekt im antiinflammatorischen Interleukin-10-Stoffwechselweg). Wahrscheinlich ist auch, dass das entsprechende Antigen „chronisch“ vorhanden ist, d. h. Bestandteil des dysregulierten Mikrobioms des Patienten ist. Zwar ist es durchaus zu begrüßen, dass mittlerweile eine kleine Auswahl an Biologikatherapien zur Behandlung der CED zur Verfügung steht. Jedoch sollte das in keiner Weise die Suche nach den eigentlichen Krankheitsursachen verlangsamen, zumal die Genetik der Lösung des Rätsels schon ein Stück weit entgegengekommen ist.

\section{Fazit für die Praxis}

- Chronisch-entzündliche Darmerkrankungen umfassen ein Spektrum von Entzündungsformen, in dem der Morbus Crohn mit reinem Dünndarmbefall und die Colitis ulcerosa die beiden Extreme eines fließenden Übergangs darstellen. Morbus Crohn mit reinem Befall des Dickdarms sollte als distinkte 3. Entität zwischen den beiden Extremen betrachtet werden.

- Die häufigen genetischen Suszeptibilitätsfaktoren für die CED wurden für europäischstämmige Patienten zwischenzeitlich sämtlich identifiziert, Patienten mit anderem ethnischen
Hintergrund unterscheiden sich nur gering in ihrem genetischen Risikoprofil.

- Die Aufklärung der ursächlichen genetischen Prinzipien inklusive der damit verbundenen primären pathophysiologischen Ereignisse hat die Entwicklung neuer Therapieverfahren beeinflusst.

- Die genetische Ätiologie chronischentzündlicher Darmerkrankungen ist überraschend polygen. Zudem sind viele der Krankheitsgene auch für chronische Erkrankungen relevant, die vordergründig nicht mit dem Darm assoziiert sind.

- Patienten mit einem CED-Ersterkrankungsalter $<16$ Jahre sollten eine genetische Diagnostik (seit 1.7.2016 abrechenbar) durchlaufen. Es gilt: Je jünger der Patient bei Ersterkrankung, desto wahrscheinlicher ist das Vorliegen einer monogenen Form (nur eine kausative Mutation) der CED.

\section{Korrespondenzadresse}

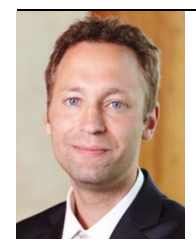

Prof. Dr. A. Franke

Institut für Klinische Molekularbiologie, ChristianAlbrechts-Universität zu Kiel Rosalind-Franklin-Str. 12, 24105 Kiel, Deutschland a.franke@mucosa.de

\section{Einhaltung ethischer Richtlinien}

Interessenkonflikt. F. Degenhardt und A. Franke geben an, dass kein Interessenkonflikt besteht.

Dieser Beitrag beinhaltet keine von den Autoren durchgeführten Studien an Menschen oder Tieren.

Open Access Dieser Artikel wird unter der Creative Commons Namensnennung 4.0 International Lizenz (http://creativecommons.org/licenses/by/4.0/deed. de) veröffentlicht, welche die Nutzung, Vervielfältigung, Bearbeitung, Verbreitung und Wiedergabe in jeglichem Medium und Format erlaubt, sofern Sie den/die ursprünglichen Autor(en) und die Quelle ordnungsgemäßnennen, einen Linkzur Creative Commons Lizenz beifügen und angeben, ob Änderungen vorgenommen wurden.

\section{Literatur}

1. Anderson CA, Boucher $G$, Lees CW, Franke $A$, D'Amato M, Taylor KD, Lee JC, Goyette P, Imielinski
M, Latiano A, Lagacé C, Scott R, Amininejad L, Bumpstead S, Baidoo L, Baldassano RN, Barclay M, Bayless TM, Brand S, Büning C, Colombel JF, Denson LA, De Vos M, Dubinsky M, Edwards C, Ellinghaus D, Fehrmann RS, Floyd JA, Florin $T$, Franchimont D, Franke L, Georges M, Glas J, Glazer NL, Guthery SL, Haritunians T, Hayward NK, Hugot JP, Jobin G, Laukens D, Lawrance I, Lémann $M$, Levine A, Libioulle C, Louis E, McGovern DP, Milla M, Montgomery GW, Morley KI, Mowat C, Ng A, Newman W, Ophoff RA, Papi L, Palmieri O, PeyrinBiroulet L, Panés J, Phillips A, Prescott NJ, Proctor $D D$, Roberts R, Russell R, Rutgeerts $P$, Sanderson J, Sans M, Schumm P, Seibold F, Sharma Y, Simms LA, Seielstad M, Steinhart AH, Targan SR, van den Berg $L H$, Vatn $M$, Verspaget $H$, Walters $T$, Wijmenga C, Wilson DC, Westra HJ, Xavier RJ, Zhao ZZ, Ponsioen CY, Andersen V, Torkvist L, Gazouli M, Anagnou NP, Karlsen TH, Kupcinskas L, Sventoraityte J, Mansfield JC, Kugathasan S, Silverberg MS, Halfvarson J, Rotter Jl, Mathew CG, Griffiths AM, Gearry R, Ahmad T, Brant SR, Chamaillard M, Satsangi J, Cho JH, Schreiber S, Daly MJ, Barrett JC, Parkes M, Annese V, Hakonarson H, Radford-Smith G, Duerr RH, Vermeire S, Weersma RK, Rioux JD (2011) Meta-analysis identifies 29 additional ulcerative colitis risk loci, increasing the number of confirmed associations to 47 . Nat Genet 43(3):246-252. doi:10.1038/ng.764

2. Barrett JC, Hansoul S, Nicolae DL, Cho JH, Duerr $\mathrm{RH}$, Rioux JD, Brant SR, Silverberg MS, Taylor KD, Barmada MM, Bitton A, Dassopoulos T, Datta LW, Green T, Griffiths AM, Kistner EO, Murtha MT, Regueiro MD, Rotter Jl, Schumm LP, Steinhart AH, Targan SR, Xavier RJ, NIDDK IBD Genetics Consortium, Libioulle $C$, Sandor C, Lathrop M, Belaiche J, Dewit O, Gut I, Heath S, Laukens D, Mni M, Rutgeerts P, Van Gossum A, Zelenika D, Franchimont $D$, Hugot JP, de Vos $M$, Vermeire $S$, Louis E, Wellcome Trust Case Control Consortium, Belgian-French IBD Consortium, Cardon LR, Anderson CA, Drummond H, Nimmo E, Ahmad T, Prescott NJ, Onnie CM, Fisher SA, Marchini J, Ghori J, BumpsteadS, Gwilliam R, Tremelling M, Deloukas P, Mansfield J, Jewell D, Satsangi J, Mathew CG, Parkes M, Georges M, Daly MJ (2008) Genomewide association defines more than 30 distinct susceptibility loci for Crohn's disease. Nat Genet 40(8):955-962. doi:10.1038/ng.175

3. Beaudoin M, Goyette P, Boucher G, Lo KS, Rivas MA, Stevens C, Alikashani A, Ladouceur M, Ellinghaus D, Törkvist L, Goel G, Lagacé $C$, Annese V, Bitton A, Begun J, Brant SR, Bresso F, Cho JH, Duerr $\mathrm{RH}$, Halfvarson J, McGovern DP, Radford-Smith G, Schreiber S, Schumm PL, Sharma Y, Silverberg MS, Weersma RK, Quebec IBD Genetics Consortium, NIDDK IBD Genetics Consortium, International IBD Genetics Consortium, D'Amato M, Vermeire $S$, Franke A, Lettre G, Xavier RJ, Daly MJ, Rioux JD (2013) Deep resequencing of GWAS loci identifies rare variants in CARD9, IL23R and RNF186 that are associated with ulcerative colitis. PLOS Genet 9(9):e1003723. doi:10.1371/journal.pgen. 1003723

4. Brant SR (2011) Update on the heritability of inflammatory bowel disease: the importance of twin studies. Inflamm Bowel Dis 17(1):1-5. doi:10. 1002/ibd. 21385

5. Cleynen I, Boucher G, Jostins L, Schumm LP, Zeissig $\mathrm{S}$, Ahmad T, Andersen V, Andrews JM, Annese V, Brand S, Brant SR, Cho JH, Daly MJ, Dubinsky $M$, Duerr RH, Ferguson LR, Franke A, Gearry RB, Goyette P, Hakonarson $\mathrm{H}$, Halfvarson J, Hov JR, Huang $H$, Kennedy NA, Kupcinskas L, Lawrance 
IC, Lee JC, Satsangi J, Schreiber S, Théâtre E, van der Meulen-de Jong AE, Weersma RK, Wilson DC, International Inflammatory Bowel Disease Genetics Consortium, Parkes M, Vermeire S, Rioux JD, Mansfield J, Silverberg MS, Radford-Smith G, McGovern DP, Barrett JC, Lees CW (2016) Inherited determinants of Crohn's disease and ulcerative colitis phenotypes: a genetic association study. Lancet 387(10014):156-167. doi:10.1016/S01406736(15)00465-1

6. Duerr RH, Taylor KD, Brant SR, Rioux JD, Silverberg MS, Daly MJ, Steinhart AH, Abraham C, Regueiro M, Griffiths A, Dassopoulos T, Bitton A, Yang $H$, Targan S, Datta LW, Kistner EO, Schumm LP, Lee AT, Gregersen PK, Barmada MM, Rotter Jl, Nicolae DL, Cho JH (2006) A genome-wide association study identifies IL23R as an inflammatory bowel disease gene. Science 314(5804):1461-1463

7. Ellinghaus D, Jostins L, Spain SL, Cortes A, Bethune J, Han B, Park YR, Raychaudhuri S, Pouget JG, Hübenthal M, Folseraas T, Wang Y, Esko T, Metspalu A, Westra HJ, Franke L, Pers TH, Weersma RK, Collij V, D'Amato $M$, Halfvarson J, Jensen $A B$, Lieb W, Degenhardt F, Forstner AJ, Hofmann A, International IBD Genetics Consortium (IIBDGC), International Genetics of Ankylosing Spondylitis Consortium (IGAS), International PSC Study Group (IPSCSG), Genetic Analysis of Psoriasis Consortium (GAPC), Psoriasis Association Genetics Extension (PAGE), Schreiber S, Mrowietz U, Juran BD, Lazaridis KN, Brunak S, Dale AM, Trembath RC, Weidinger S, Weichenthal M, Ellinghaus E, Elder JT, Barker JN, Andreassen OA, McGovern DP, Karlsen TH, Barrett JC, Parkes M, Brown MA, Franke A (2016) Analysis of five chronic inflammatory diseases identifies 27 new associations and highlights disease-specific patterns at shared loci. Nat Genet 48(5):510-518. doi:10.1038/ng.3528

8. Farh KK, Marson A, Zhu J, Kleinewietfeld M, Housley WJ, Beik S, Shoresh N, Whitton H, Ryan RJ, Shishkin AA, Hatan M, Carrasco-Alfonso MJ, Mayer D, Luckey CJ, Patsopoulos NA, De Jager PL, Kuchroo VK, Epstein CB, Daly MJ, Hafler DA, Bernstein BE (2015) Genetic and epigenetic fine mapping of causal autoimmune disease variants. Nature 518(7539):337-343. doi:10.1038/nature13835

9. Feller M, Huwiler K, Stephan R, Altpeter E, Shang A, Furrer H, Pfyffer GE, Jemmi T, Baumgartner A, Egger M (2007) Mycobacterium avium subspecies paratuberculosis and Crohn's disease: a systematic review and meta-analysis. Lancet Infect Dis 7(9):607-613

10. Franke A, Balschun $T$, Karlsen $T H$, Sventoraityte J, Nikolaus S, Mayr G, Domingues FS, Albrecht $M$, Nothnagel $M$, Ellinghaus $D$, Sina $C$, Onnie CM, Weersma RK, Stokkers PC, Wijmenga C, Gazouli M, Strachan D, McArdle WL, Vermeire S, Rutgeerts P, Rosenstiel P, Krawczak M, Vatn MH, BSEN study group, Mathew CG, Schreiber S (2008) Sequence variants in IL10, ARPC2 and multiple other loci contribute to ulcerative colitis susceptibility. Nat Genet 40(11):1319-1323. doi:10.1038/ng.221

11. Franke A, McGovern DP, Barrett JC, Wang K, Radford-Smith GL, Ahmad T, Lees CW, Balschun T, Lee J, Roberts R, Anderson CA, Bis JC, Bumpstead S, Ellinghaus D, Festen EM, Georges M, Green T, Haritunians T, Jostins L, Latiano A, Mathew CG, Montgomery GW, Prescott NJ, Raychaudhuri S, Rotter JI, Schumm P, Sharma Y, Simms LA, Taylor KD, Whiteman D, Wijmenga C, Baldassano RN, Barclay M, Bayless TM, Brand S, Büning C, Cohen A, Colombel JF, Cottone M, Stronati L, Denson T, De Vos M, D'Inca R, Dubinsky M, Edwards C, Florin T, Franchimont D, Gearry R, Glas J, Van Gossum
A, Guthery SL, Halfvarson J, Verspaget HW, Hugot JP, Karban A, Laukens D, Lawrance I, Lemann M, Levine A, Libioulle C, Louis E, Mowat C, Newman W, Panés J, Phillips $A$, Proctor DD, Regueiro $M$, Russell R, Rutgeerts P, Sanderson J, Sans M, Seibold F, Steinhart AH, Stokkers PC, Torkvist L, Kullak-Ublick G, Wilson D, Walters T, Targan SR, Brant SR, Rioux JD, D'Amato M, Weersma RK, Kugathasan S, Griffiths AM, Mansfield JC, Vermeire S, Duerr RH, Silverberg MS, Satsangi J, Schreiber S, Cho JH, Annese V, Hakonarson H, Daly MJ, Parkes M (2010) Genomewide meta-analysis increases to 71 the number of confirmed Crohn's disease susceptibility loci. Nat Genet 42(12):1118-1125. doi:10.1038/ng.717

12. GoyetteP,BoucherG, Mallon D, EllinghausE, Jostins L, Huang H, Ripke S, Gusareva ES, Annese V, Hauser SL, Oksenberg JR, Thomsen I, Leslie S, International InflammatoryBowelDisease Genetics Consortium, Australia and New Zealand IBDGC, Belgium IBD Genetics Consortium, Italian Group for IBD Genetic Consortium, NIDDK Inflammatory Bowel Disease Genetics Consortium, United Kingdom IBDGC, Wellcome Trust Case Control Consortium; Quebec IBD Genetics Consortium, Daly MJ, Van Steen K, Duerr RH, Barrett JC, McGovern DP, Schumm $\mathrm{LP}$, Traherne JA, Carrington MN, Kosmoliaptsis V, Karlsen TH, Franke A, Rioux JD, Quebec IBD Genetics Consortium, Daly MJ, Van Steen K, Duerr $\mathrm{RH}$, Barrett JC, McGovern DP, Schumm LP, Traherne JA, Carrington MN, Kosmoliaptsis V, Karlsen TH, Franke A, Rioux JD (2015) High-density mapping of the MHC identifies a shared role for HLADRB1*01:03 in inflammatory bowel diseases and heterozygous advantage in ulcerative colitis. Nat Genet 47(2):172-179. doi:10.1038/ng.3176

13. Halme L, Paavola-Sakki P, Turunen U, Lappalainen M, Farkkila M, Kontula K (2006) Family and twin studies in inflammatory bowel disease. World J Gastroenterol 12(23):3668-3672

14. Hampe J, Franke A, Rosenstiel P, Till A, Teuber M, Huse K, Albrecht M, Mayr G, De La Vega FM, Briggs J, Günther S, Prescott NJ, Onnie CM, Häsler R, Sipos B, Fölsch UR, Lengauer T, Platzer M, Mathew CG, Krawczak M, Schreiber S (2007) A genomewide association scan of nonsynonymous SNPs identifies a susceptibility variant for $C$ rohn disease in ATG16L1. Nat Genet 39(2):207-211

15. Hugot JP, Laurent-Puig P, Gower-Rousseau C, Olson JM, Lee JC, Beaugerie L, Naom I, Dupas JL, Van Gossum A, Orholm M, Bonaiti-Pellie C, Weissenbach J, Mathew CG, Lennard-Jones JE, Cortot A, Colombel JF, Thomas G (1996) Mapping of a susceptibility locus for Crohn's disease on chromosome 16. Nature 379(6568):821-823

16. Hugot JP, Chamaillard $M$, Zouali $H$, Lesage $S$, Cézard JP, Belaiche J, Almer S, Tysk C, O'Morain CA Gassull M, Binder V, Finkel Y, Cortot A, Modigliani R, Laurent-Puig P, Gower-Rousseau C, Macry J, Colombel JF, Sahbatou M, Thomas G (2001) Association of NOD2 leucine-rich repeat variants with susceptibility to Crohn's disease. Nature 411(6837):599-603

17. Jostins L, RipkeS, WeersmaRK, DuerrRH,McGovern DP, Hui KY, Lee JC, Schumm LP, Sharma Y, Anderson CA, Essers J, Mitrovic M, Ning K, Cleynen I, Theatre E, Spain SL, Raychaudhuri S, Goyette P, Wei Z, Abraham C, Achkar JP, Ahmad T, Amininejad L, Ananthakrishnan AN, Andersen V, Andrews JM, Baidoo L, Balschun T, Bampton PA, Bitton A, Boucher G, Brand S, Büning C, Cohain A, Cichon S, D'Amato M, De Jong D, Devaney KL, Dubinsky M, Edwards $C$, Ellinghaus $D$, Ferguson $L R$, Franchimont D, Fransen K, Gearry R, Georges M, Gieger C, Glas J, Haritunians T, Hart A, Hawkey C, Hedl M,
Hu X, Karlsen TH, Kupcinskas L, Kugathasan S, Latiano A, Laukens D, Lawrance IC, Lees CW, Louis E, Mahy G, Mansfield J, Morgan AR, Mowat C, Newman W, Palmieri O, Ponsioen CY, Potocnik U, Prescott NJ, Regueiro M, Rotter J, Russell RK, Sanderson JD, Sans M, Satsangi J, Schreiber S, Simms LA, Sventoraityte J, Targan SR, Taylor KD, Tremelling M, Verspaget HW, De Vos M, Wijmenga C, Wilson DC, Winkelmann J, Xavier RJ, Zeissig $\mathrm{S}$, Zhang B, Zhang CK, Zhao H, International IBD Genetics Consortium (IIBDGC), Silverberg MS, Annese V, Hakonarson H, Brant SR, Radford-Smith G, Mathew CG, Rioux JD, Schadt EE, Daly MJ, Franke A, Parkes M, Vermeire S, Barrett JC, Cho $\mathrm{JH}$ (2012) Host-microbe interactions have shaped the genetic architecture of inflammatory bowel disease. Nature 491(7422):119-124. doi:10.1038/ nature 11582

18. Kaplan GG (2015) The global burden of IBD: from 2015 to 2025. Nat Rev Gastroenterol Hepatol 12(12):720-727. doi:10.1038/nrgastro.2015.150

19. Karlsen TH, Chung BK (2015) Genetic risk and the development of autoimmune liver disease. Dig Dis 33(Suppl 2):13-24. doi:10.1159/000440706

20. Lees CW, Barrett JC, Parkes M, Satsangi J (2011) New IBD genetics: common pathways with other diseases. Gut 60(12):1739-1753. doi:10.1136/gut. 2009.199679

21. Liu JZ, van Sommeren S, Huang $\mathrm{H}, \mathrm{Ng}$ SC, Alberts R, Takahashi A, Ripke S, Lee JC, Jostins L, Shah T, Abedian S, Cheon JH, Cho J, Daryani NE, Franke L, Fuyuno Y, Hart A, Juyal RC, Juyal G, Kim WH, Morris AP, Poustchi H, Newman WG, Midha V, Orchard TR, Vahedi H, Sood A, Sung JJ, Malekzadeh R, Westra HJ, Yamazaki K, Yang SK, International Multiple Sclerosis Genetics Consortium, International IBD Genetics Consortium, Barrett JC, Franke A, Alizadeh BZ, Parkes M, Daly MJ, Kubo M, Anderson CA, Weersma RK (2015) Association analyses identify 38 susceptibility loci for inflammatory bowel disease and highlight shared genetic risk across populations. Nat Genet 47(9):979-986. doi:10.1038/ng.3359

22. Mathew CG, Lewis CM (2004) Genetics of inflammatory bowel disease: progress and prospects. Hum Mol Genet 13(1):R161-R168

23. McGovern DP, Jones MR, Taylor KD, Marciante K, Yan X, Dubinsky M, Ippoliti A, Vasiliauskas E, Berel D, Derkowski C, Dutridge D, Fleshner P, Shih DQ, Melmed G, Mengesha E, King L, Pressman S, Haritunians T, Guo X, Targan SR, Rotter JI, International IBD Genetics Consortium (2010) Fucosyltransferase 2 (FUT2) non-secretor status is associated with Crohn's disease. Hum Mol Genet 19(17):3468-3476. doi:10.1093/hmg/ddq248

24. Muñoz M, Pong-Wong R, Canela-Xandri O, Rawlik K, Haley CS, Tenesa A (2016) Evaluating the contribution of genetics and familial shared environment to common disease using the UK Biobank. Nat Genet 48(9):980-983. doi:10.1038/ ng.3618

25. Ogura Y, Bonen DK, Inohara N, Nicolae DL, Chen FF, Ramos R, Britton H, Moran T, Karaliuskas R, Duerr RH, Achkar JP, Brant SR, Bayless TM, Kirschner BS, Hanauer SB, Nuñez G, Cho JH (2001) A frameshift mutation in NOD2 associated with susceptibility to Crohn's disease. Nature 411(6837):603-606

26. Parkes M, Cortes A, van Heel DA, Brown MA (2013) Genetic insights into common pathways and complex relationships among immune-mediated diseases. Nat Rev Genet 14(9):661-673. doi:10. 1038/nrg3502 
27. Risch N, Merikangas K (1996) The future of genetic studies of complex human diseases. Science 273(5281):1516-1517

28. Rivas MA, Beaudoin M, Gardet A, Stevens C, Sharma $Y$, Zhang CK, Boucher G, Ripke S, Ellinghaus D, Burtt N, Fennell T, Kirby A, Latiano A, Goyette $P$, Green T, Halfvarson J, Haritunians T, Korn JM, Kuruvilla F, Lagacé C, Neale B, Lo KS, Schumm $P$, Törkvist L, National Institute of Diabetes and Digestive Kidney Diseases Inflammatory Bowe Disease Genetics Consortium (NIDDK IBDGC), United Kingdom Inflammatory Bowel Disease Genetics Consortium, International Inflammatory Bowel Disease Genetics Consortium, Dubinsky MC Brant SR, Silverberg MS, Duerr RH, Altshuler D, Gabriel S, Lettre G, Franke A, D'Amato M, McGovern DP, Cho JH, Rioux JD, Xavier PJ, Daly MJ (2011) Deep resequencing of GWAS loci identifies independent rare variants associated with inflammatory bowel disease. Nat Genet 43(11):1066-1073. doi:10. 1038/ng.952

29. Tschurtschenthaler M, Kachroo $P$, Heinsen FA, Adolph TE, Rühlemann MC, Klughammer J, Offner FA, Ammerpohl O, Krueger F, Smallwood S, Szymczak S, Kaser A, Franke A (2016) Paternal chronic colitis causes epigenetic inheritance of susceptibility to colitis. Sci Rep 6:31640. doi:10. 1038/srep31640

30. Uhlig HH, Schwerd T, Koletzko S, Shah N, Kammermeier J, Elkadri A, Ouahed J, Wilson DC, Travis SP, Turner D, Klein C, Snapper SB, Muise AM, COLORS in IBD Study Group and NEOPICS (2014) The diagnostic approach to monogenic very early onset inflammatory bowel disease. Gastroenterology 147(5):990-1007.e3. doi:10. 1053/j.gastro.2014.07.023

31. van Heel DA, Fisher SA, Kirby A, Daly MJ, Rioux JD, Lewis CM, Genome Scan Meta-Analysis Group of the IBD International Genetics Consortium (2004) Inflammatory bowel disease susceptibility loci defined by genome scan meta-analysis of 1952 affected relative pairs. Hum Mol Genet 13(7):763-770

32. Visscher PM, Brown MA, McCarthy MI, Yang J (2012) Five years of GWAS discovery. Am J Hum Genet 90(1):7-24. doi:10.1016/j.ajhg.2011.11.029

33. Wellcome Trust Case Control Consortium (2007) Genome-wide association study of 14,000 cases of seven common diseases and 3,000 shared controls. Nature 447(7145):661-678

34. Yamazaki K, McGovern D, Ragoussis J, Paolucci M, Butler $\mathrm{H}$, Jewell D, Cardon L, Takazoe M, Tanaka T, Ichimori T, Saito S, Sekine A, lida A, Takahashi A, Tsunoda T, Lathrop M, Nakamura Y (2005) Single nucleotide polymorphisms in TNFSF15 confer susceptibility to Crohn's disease. Hum Mol Genet 14(22):3499-3506 (Nov)

35. Zhang FR, Huang W, Chen SM, Sun LD, Liu H, Li Y, Cui Y, Yan XX, Yang HT, Yang RD, Chu TS, Zhang C, Zhang L, Han JW, Yu GQ, Quan C, Yu YX, Zhang Z, Shi BQ, Zhang $\mathrm{LH}$, Cheng $\mathrm{H}$, Wang $\mathrm{CY}$, Lin $\mathrm{Y}$, Zheng $H F$, Fu XA, Zuo XB, Wang Q, Long $H$, Sun YP, Cheng YL, Tian HQ, Zhou FS, Liu HX, Lu WS, He SM, Du WL, Shen $M$, Jin QY, Wang Y, Low HQ, Erwin T, Yang NH, Li JY, Zhao X, Jiao YL, Mao LG, Yin G, Jiang ZX, Wang XD, Yu JP, Hu ZH, Gong CH, Liu YQ, Liu RY, Wang DM, Wei D, Liu JX, Cao WK, Cao HZ, Li YP, Yan WG, Wei SY, Wang KJ, Hibberd ML, Yang S, Zhang XJ, Liu JJ (2009) Genomewide association study of leprosy. N Engl J Med 361(27):2609-2618. doi:10. 1056/NEJMoa0903753
Mitteldeutsche Gesellschaft für Gastroenterologie
Hessen - Sachsen - Sachsen-Anhalt - Thüringen

\section{Ausschreibung des Förderpreises der Mitteldeutschen Gesellschaft für Gastroenterologie e.V.}

Die Mitteldeutsche Gesellschaft für Gastroenterologie e.V. (MGG) vergibt auf ihrem 26. Jahreskongress in Frankfurt a.M. 2017 wieder einen Förderpreis für junge Kliniker und Wissenschaftler.

Der Preis wird für Forschungsarbeiten von Klinikern und Wissenschaftlern, die in den Mitgliedsländern der MGG (Hessen, Thüringen, Sachsen, Sachsen-Anhalt) tätig und unter 45 Jahre alt sind, vergeben und ist mit $3.000 €$ dotiert.

Die eingereichten Arbeiten müssen ein Forschungsthema aus dem Gebiet der klinischen Gastroenterologie behandeln und dürfen nicht länger als ein Jahr vor Ablauf der Ausschreibung fertig gestellt worden sein. Sie sollen außerdem erstmals und nicht bereits zu anderen Wettbewerben eingereicht worden sein. Die Arbeiten müssen auf eigenen wissenschaftlichen Untersuchungen beruhen, die in der Hauptsache in den Mitgliedsländern der MGG durchgeführt wurden.

Alle an der Durchführung der Untersuchungen beteiligten Mitarbeiter sind als Co-Autoren der Arbeit namentlich zu benennen und sollen ihr Einverständnis zur Teilnahme am Wettbewerb schriftlich erklären. Damit erkennen sie auch an, dass die Arbeit nicht von einem der Co-Autoren an anderer Stelle eingereicht wird. Ein wissenschaftliches Curriculum vitae sowie ein Votum informativum des Leiters der Einrichtung (Direktor/Chefarzt) sind den Bewerbungsunterlagen beizufügen.

Die Arbeiten sind in deutscher oder englischer Sprache zu 5 Exemplaren bis zum

24. März 2017 an folgende Adresse einzureichen:

Prof. Dr. med. Joachim Glaser

Schriftführer der MGG

Vitalisklinik Bad Hersfeld GmbH

Am Weinberg 3

36251 Bad Hersfeld 